Polymer Journal, Vol. 39, No. 6, pp. 509-513 (2007)

(C) 2007 The Society of Polymer Science, Japan

\title{
Fluctuation Effects on Viscoelastic Properties of Diblock Copolymer Solutions in Disordered State
}

\author{
Yoshiaki TAKAHASHI, ${ }^{1, \dagger}$ Yuki OJIKA, ${ }^{2}$ Yoshihiko AKAZAwA, ${ }^{2}$ \\ Atsushi TAKANo, ${ }^{2}$ and Yushu MATSUSHITA ${ }^{2}$ \\ ${ }^{1}$ Institute for Materials Chemistry and Engineering, and Department of Molecular \& Materials Sciences, \\ IGSES, Kyushu University, 6-1 Kasugakoen, Kasuga 816-8580, Japan \\ ${ }^{2}$ Department of Applied Chemistry, Nagoya University, Nagoya 464-8603, Japan
}

(Received November 13, 2006; Accepted March 1, 2007; Published April 17, 2007)

\begin{abstract}
Viscoelastic properties of three poly(styrene- $\mathrm{d}_{8}$-block-isoprene)s in dioctyl phthalate are measured under oscillatory and steady shear flows in the disordered state, to examine the fluctuation effects on zero shear viscosity $\eta^{0}$ and steady state compliance $J_{\mathrm{e}}$ based on the effective interaction parameter $(\chi N)_{\text {eff }}$. Flow-induced microphase separation is not observed when $(\chi N)_{\text {eff }}$ is lower than 9.9. Enhancement of $\eta^{0}$ and $J_{\mathrm{e}}$ due to the fluctuation effects, which is more obvious for $J_{\mathrm{e}}$, are observed in oscillatory flow, while suppression of the fluctuation effects are observed in steady shear flow, which is confirmed by small angle neutron scattering measurements. Comparing these results with those for poly(styrene-block-2-vinylpyridine)s (SP), it is concluded that the viscosity enhancement due to the fluctuation effects slightly depend on component viscosities. When viscosity ratio of components is close to 1 (i.e., SP), the viscosity enhancement is negligible. On the other hand, enhancement of reduced steady state compliance $J_{\mathrm{eR}}$ seems to be insensitive to the viscosity ratio of components. [doi:10.1295/polymj.PJ2006157]

KEY WORDS Poly(styrene-block-isoprene)s / Viscoelastic Properties / Disordered State / Fluctuation Effects / Small Angle Neutron Scattering /
\end{abstract}

In previous papers, ${ }^{1-5}$ we studied flow-induced structures and related viscoelastic properties of lamellae-forming diblock copolymers, that is, poly(styreneblock-2-vinylpyridine)s (SP) and poly(styrene- $\mathrm{d}_{8}$ block-2-vinylpyridine)s (DP) in a common good solvent, $\alpha$-chloronaphtharene $(\alpha-\mathrm{CN})$ and poly(styreneblock-isoprene)s (SI) and poly(styrene- $\mathrm{d}_{8}$-block-isoprene)s (DI) in dioctyl phthalate (DOP) near the order-disorder transition (ODT) under shear flows. A characteristic feature of SPs and DPs compared with SIs and DIs, and also with other common diblock copolymers is that the two component polymers have quite similar viscoelastic properties. ${ }^{6}$

Flow-induced alignments of lamellae for the DP and DI solutions are examined by small angle neutron scattering under steady shear flow (flow-SANS) at almost the same experimental conditions. ${ }^{5}$ For the DI solutions, it is observed that parallel and perpendicular alignments of lamellae, in which lamellar normals are parallel to shear gradient and vorticity directions, respectively, coexist at low shear rates $(\dot{\gamma})$ and the latter become dominant at high $\dot{\gamma}$. These results are qualitatively consistent with those reported in literatures. ${ }^{7-13}$ For the DP solutions, coexistence of the parallel and perpendicular alignments is only observed at low $\dot{\gamma}$ for relatively high polymer concentration $(C)$ above the quiescent ODT concentration, $C_{\mathrm{ODT}},{ }^{2}$ while only the perpendicular alignment was observed at lower $C$ near the $C_{\mathrm{ODT}} \cdot{ }^{3-5}$ It was concluded that the degree of perpendicular alignment is primarily determined by experimentally determined values of $\chi N,(\chi N)_{\text {eff }}$, and reduced shear rate $\dot{\gamma} / \dot{\gamma}^{*}$. Here, $\chi$ and $N$ are the Flory-Huggins interaction parameter and the number of statistical segments per copolymer molecules, respectively, and $\dot{\gamma}^{*}$ is the characteristic $\dot{\gamma}$ for non-Newtonian behavior. ${ }^{5}$ At the same $(\chi N)_{\text {eff }}$ value, the ratio of perpendicular/parallel alignments is higher for the samples whose viscosity ratio of component is close to 1 .

In the disordered states close to the ODT $\left(C_{\mathrm{ODT}}>\right.$ $C \geq C_{\mathrm{ODT}}-1.5 \mathrm{wt} \%$ ), flow-induced ordering with the perpendicularly aligned lamellae are observed for the DI solutions, ${ }^{5}$ whereas no flow-induced ordering but suppression of fluctuation effects is observed for a DP solution only $0.5 \mathrm{wt} \%$ below the $C_{\mathrm{ODT}}{ }^{4}$ The difference can be attributed to the difference in the magnitude of fluctuation effects. That is, $(\chi N)_{\text {eff }}$ for the tested DP solution is slightly lower than that for DI solutions. Actually, suppression of fluctuation is also observed for DI solution having almost the same $(\chi N)_{\text {eff }}$ value as the above mentioned DP solution, though the 2 dimensional scattering pattern of flow-SANS was still slightly anisotropic. ${ }^{5}$

It was pointed out from the rheological measurements ${ }^{1,4}$ that the first normal stress difference $N_{1}$ of diblock copolymer solutions in the ordered states are

${ }^{\dagger}$ To whom correspondence should be addressed (Tel/Fax: +81-92-583-8822, E-mail: ytak@mm.kyushu-u.ac.jp). 
proportional to $\dot{\gamma}$ in low $\dot{\gamma}$ regime. In relatively high $\dot{\gamma}$ regime, where the lamellae are well aligned, shear stress $\sigma$ and $N_{1}$ are proportional to $\dot{\gamma}$ and $\dot{\gamma}^{2}$, respectively, so that zero shear viscosity $\eta^{0}$ and steady state compliance $J_{\mathrm{e}}$ can be obtained by ordinary methods. The values of $\eta^{0}$ and $J_{\mathrm{e}}$ for DP and SP solutions thus obtained $^{4}$ are practically the same as those of corresponding component homopolymers. ${ }^{6}$

In the disordered states of SP and DP solutions, $\eta^{0}$ data are also practically the same as those of corresponding components, implying that enhancement of viscosity due to fluctuation effects is negligible for these samples. On the other hand, $J_{\mathrm{e}}$ obtained by dynamic viscoelastic measurements are higher than those of corresponding components due to the fluctuation effects, but the $J_{\mathrm{e}}$ data become almost the same as those of components under steady shear flow because of suppression of the fluctuation effects, confirmed by flow-SANS measurements. ${ }^{4}$

Rather strong fluctuation effects are reported for poly(ethylenepropyrene-block-ethylethyrene) ${ }^{14}$ and $\mathrm{SI},{ }^{15}$ in which storage modulus is much affected than loss modulus. Thus, above mentioned differences in $\eta^{0}$ and $J_{\mathrm{e}}$ for SP and DP is qualitatively consistent with those studies, though the viscosity enhancement is not observed even in oscillatory flow measurement.

In this study, we further examine fluctuation effects on $\eta^{0}$ and $J_{\mathrm{e}}$ and their suppressions under steady shear flow. The data for three different DI sample solutions in the disordered states measured under oscillatory and steady shear flow are compared. Flow-SANS measurements are also carried out for a few selected conditions to examine the structure.

\section{EXPERIMENTAL}

Diblock copolymer samples used are three different poly(styrene- $\mathrm{d}_{8}-$-block-isoprene)s (DI) prepared by anionic polymerization method. Number-averaged molecular weight, $M_{\mathrm{n}}$, weight-averaged molecular weight, $M_{\mathrm{w}}$, molecular weight distribution index, $M_{\mathrm{w}} / M_{\mathrm{n}}$, and volume fraction of styrene- $\mathrm{d}_{8}, \phi_{\mathrm{PS}}$, are determined by combination of osmotic pressure, ${ }^{1} \mathrm{H}$ NMR and GPC measurements as in the previous paper. ${ }^{5}$ Molecular characteristics of the samples are tabulated in Table I. The solvent used is DOP and all the measurements mentioned below are carried out at $27.0 \pm 0.5^{\circ} \mathrm{C}$.

Table I. Molecular Characteristics of DI Samples

\begin{tabular}{ccccc}
\hline Sample & $10^{-4} M_{\mathrm{w}}$ & $M_{\mathrm{w}} / M_{\mathrm{n}}$ & $\phi_{\mathrm{PS}}$ & $C_{\text {ODT }}(\mathrm{wt} \%)$ \\
\hline DI-55 & $1.4_{6}$ & 1.04 & 0.5 & $>70$ \\
DI-15 & $3.9_{9}$ & 1.05 & 0.5 & 50 \\
DI-10 & 11.5 & 1.09 & 0.5 & 27.5 \\
\hline
\end{tabular}

To obtain interaction parameters and to examine the structure, ordinary SANS and flow-SANS measurements are carried out by SANS-U spectrometer at the Neutron Scattering Laboratory of the ISSP, The University of Tokyo in Tokai, Ibaraki, Japan. The wavelength $\lambda$ and beam size of the incident neutrons are $0.7 \mathrm{~nm}$ and $3 \mathrm{~mm} \phi$, respectively. Sample to detector distance is $4 \mathrm{~m}$. Ordinary quartz cell with sample thickness of $2 \mathrm{~mm}$ is used to determine the ODT condition and $(\chi N)_{\text {eff }}$, while a couette type flow cell ${ }^{16}$ is used for flow-SANS measurements. Through view configuration, in which the incident neutron beam is in the direction normal to the surface of the center part of couette cell (along the shear gradient direction) is employed in the flow-SANS measurements. The SANS data are corrected for the incoherent scattering of the solvent and the empty cell. Circularly averaged intensities $I(q)$ are obtained as a function of wave vector, $q$, defined by $q=4 \pi \sin (\theta / 2) / \lambda$, where $\theta$ is scattering angle. Note that the flow-SANS data are also discussed by $I(q)$ since the scattering pattern was always isotropic.

Rheological measurements are performed with Rheometrics mechanical spectrometer RMS-800. A cone-plate geometry of $5 \mathrm{~cm}$ diameter and $0.04 \mathrm{rad}$ cone-angle was used. Both dynamic and steady shear measurements are carried out for solutions with relatively higher concentrations, while only the former is carried out for the rest of solutions to obtain $\eta^{0}$.

\section{RESULTS AND DISCUSSIONS}

As already discussed in the previous work, ${ }^{5}$ peak intensity of SANS measurements depends on the alignment of lamellae in the ordered states so that the full width at half maximum values of $I(q)$ vs. $q$ plots are used to determine $C_{\mathrm{ODT}}$ for DI-10. In this work too, $C_{\mathrm{ODT}}$ for DI- 15 is determined by the same method. $C_{\mathrm{ODT}}$ at $27^{\circ} \mathrm{C}$ for these samples are also shown in Table I. It is expected that $C_{\mathrm{ODT}}$ for DI-55 at $27{ }^{\circ} \mathrm{C}$ is close to bulk state since its molecular weight is very low. Thus, it is only confirmed by SANS pattern that DI-55 is in the disordered state at $70 \mathrm{wt} \%$, much higher than the concentrations for the viscoelastic measurements.

Further, the SANS data for DI-15 obtained in the disordered states are fitted to the theoretical function of Leibler ${ }^{17}$ by the same method as in the previous works ${ }^{4,5,18}$ to obtain $(\chi N)_{\text {eff }}$. Figure 1 shows plots of $(\chi N)_{\text {eff }} v s . \mathrm{C} / C_{\text {ODT }}$. The arrow in the figure indicate the highest concentration (22 wt \%) where flowinduced microphase separation was not observed for DI-10 in the previous work..$^{5}$ It is expected that the flow-induced microphase separation does not occur for the samples with lower $(\chi N)_{\text {eff }}$ values than the 


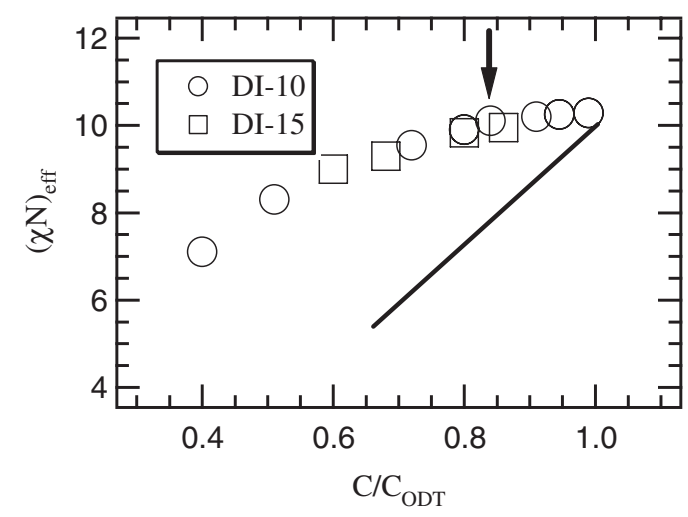

Figure 1. Plots of $(\chi N)_{\mathrm{eff}} v s . C / C_{\mathrm{ODT}}$ for DI/DOP solutions in the disordered state. Solid line represents the data for $\mathrm{DP} / \alpha$ $\mathrm{CN}$ solutions. ${ }^{5}$ Symbols are denoted in the figure. Allow indicates the highest concentration ( $22 \mathrm{wt} \%$ ) where flow-induced microphase separation was not observed for DI-10 in the previous study. ${ }^{5}$

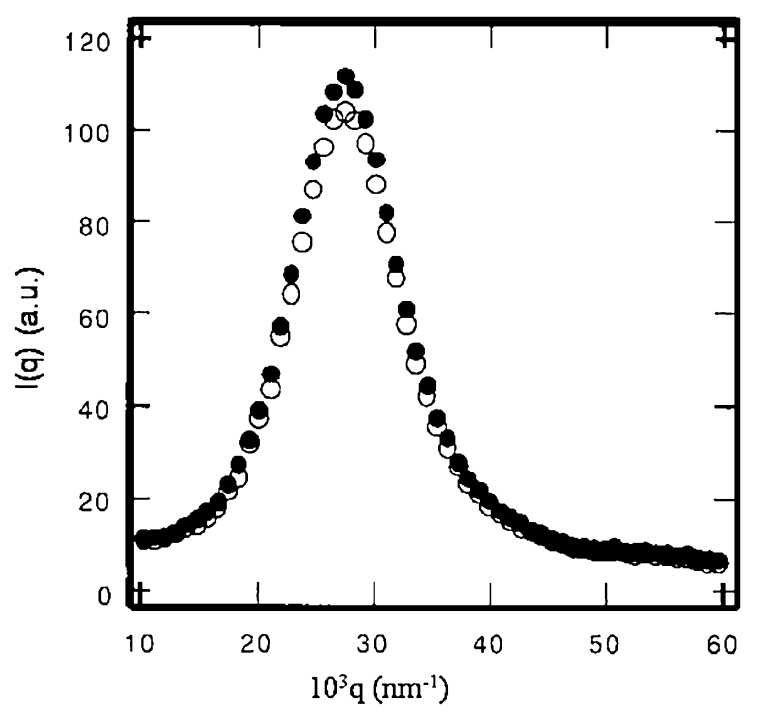

Figure 2. Plots of $I(q) v s . q$ for DI-10, $40 \mathrm{wt} \%$ solution. Filled symbol denotes the data for quiescent state while open symbol denote the data under the flow $\left(\dot{\gamma}=10 \mathrm{~s}^{-1}\right)$.

datum indicated by the arrow.

To confirm the above speculation, flow-SANS measurements are performed for DI-15 solutions at $10 \mathrm{~s}^{-1}$ for the highest two concentrations. Since the scattering data are isotropic, circularly averaged intensities $I(q)$ are obtained. Figure 2 shows plots of $I(q)$ vs. $q$ for DI-15, $40 \mathrm{wt} \%$ solution. The profile with and without shear are almost the same, but the peak intensity under the flow became about $10 \%$ lower than that at rest, indicating suppression of the fluctuations by the steady shear flow. Qualitatively the same results are observed for the other solution. We could not test at different $\dot{\gamma}$ due to limited beam time. However, the tested $\dot{\gamma}$ is about 3 order higher than the $\dot{\gamma}$ used for DI-10, $26 \mathrm{wt} \%$, in which flow induced microphase separation was observed, ${ }^{5}$ so that the above ex-
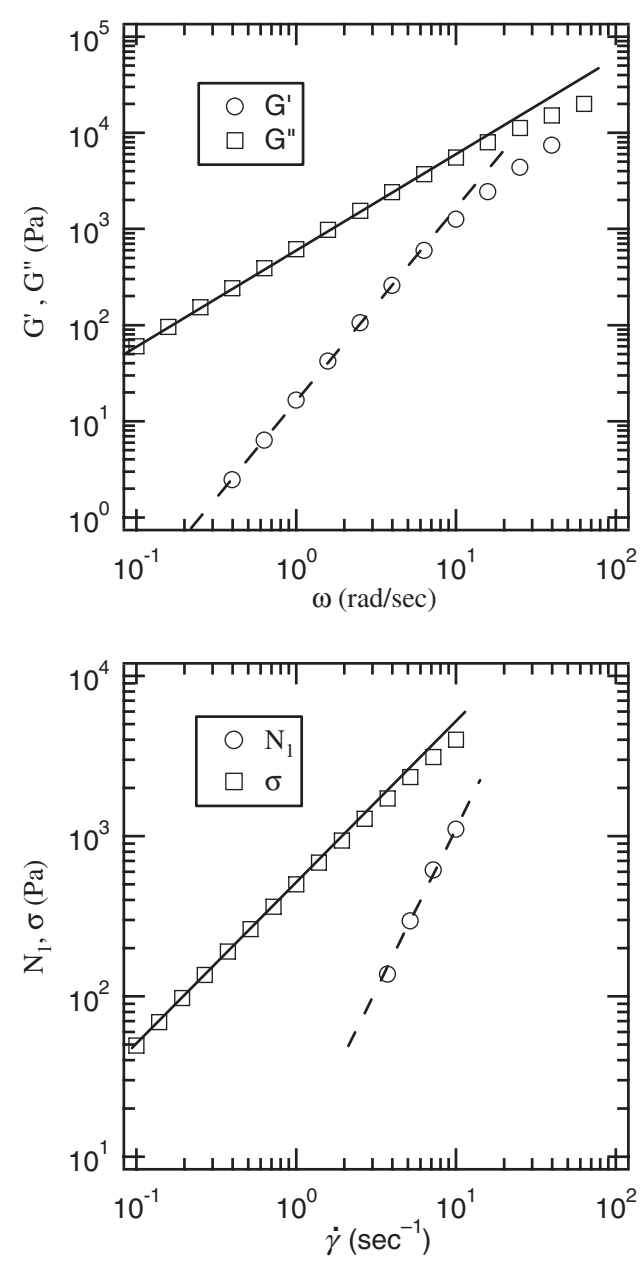

Figure 3. Double logarithmic plots of $G^{\prime}$ and $G^{\prime \prime}$ vs. $\omega$ (top) and $\sigma$ and $N_{1}$ vs. $\dot{\gamma}$ (bottom) for DI-15, $40 \mathrm{wt} \%$ solution. Solid and broken lines denote slopes of 1 and 2, respectively. Symbols are denoted in the figure.

periments are enough to conclude that flow-induced ordering does not occur at $(\chi N)_{\text {eff }}<9.9$.

Figure 3 shows examples of double logarithmic plots of storage $\left(G^{\prime}\right)$ and loss $\left(G^{\prime \prime}\right)$ moduli vs. frequency $(\omega)$ and shear stress $\sigma$ and first normal stress difference $N_{1} v s$. shear rate $\dot{\gamma}$. It is clear that $G^{\prime}$ and $G^{\prime \prime}$ are proportional to $\omega^{2}$ and $\omega$, respectively, at the lower frequency regime. It is also clear that $\sigma$ and $N_{1}$ are proportional to $\dot{\gamma}$ and $\dot{\gamma}^{2}$, respectively. From these data, we obtained zero shear viscosity $\eta^{0}$ and steady state compliance $J_{\mathrm{e}}$ by the ordinary methods.

Figure 4 shows double logarithmic plots of $\eta^{0} v s$. $C$. The data obtained by oscillatory flow measurements for DI-10, 26 and $22 \mathrm{wt} \%$ solutions and DI$15,40 \mathrm{wt} \%$ solution are slightly higher than those obtained by steady flow measurements. Flow-induced ordering was observed for DI-10, 26 wt \% solution, ${ }^{5}$ whereas flow suppression of fluctuation effects was observed for other two solutions (Figure 2). Thus, we conclude that the higher values of $\eta^{0}$ obtained by oscillatory flow measurements are due to the fluc- 


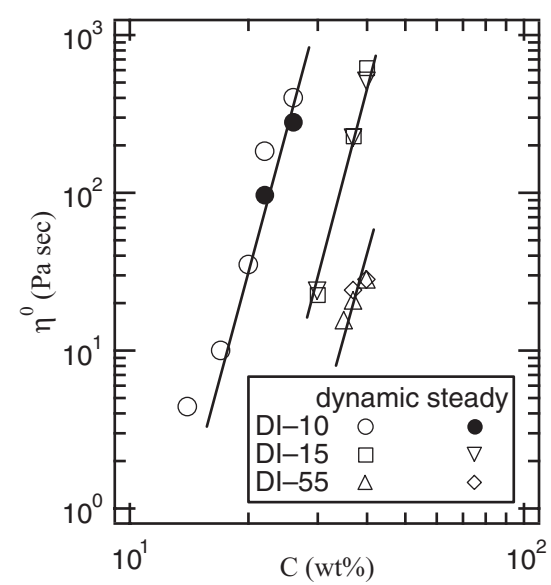

Figure 4. Double logarithmic plots of $\eta^{0} v s$. $C$ for DI/DOP solutions. Solid lines are guide for eyes. Symbols are denoted in the figure.

tuation effects. The difference between oscillatory and steady shear flow behaviors will be discussed later together with that of $J_{\mathrm{e}}$.

For the rest of DI solutions, there is no apparent difference between the data obtained from dynamic and steady flow measurements, implying that the fluctuation is not affective for those low $C$ solutions. The data obtained by steady flow measurements and dynamic measurements at lower concentrations compose respective straight lines. The slope is about 5, which is intermediate value for the concentration dependence of homopolymers in good and $\theta$ solvents ${ }^{19,20}$ and close to that for SP diblock copolymers in a selective solvent. ${ }^{21}$ Since DOP is a $\theta$ solvent for polystyrenes at $22^{\circ} \mathrm{C},{ }^{20}$ it may be a selective solvent for SI and DI, so that the observed $C$ dependence of $\eta^{0}$ may be reasonable.

Figure 5 shows double logarithmic plots of reduced steady state compliance $J_{\mathrm{eR}}\left(=J_{\mathrm{e}} C \mathrm{R} T / M_{\mathrm{W}}\right)\left[\eta^{0} /\left(\eta^{0}-\right.\right.$ $\left.\left.\left.\eta_{\mathrm{s}}\right)\right]^{2}\right) v s . C M_{\mathrm{W}}$ for DI solutions. It is clear that the data for DI-10 and DI-15 obtained by oscillatory flow are always higher than those obtained by steady flow due to the fluctuation effects, while the data for DI55 obtained by the different measurements are almost the same and close to the data of linear homopolymer systems ${ }^{22}$ since these solutions are far from the ODT. The data obtained by steady shear flow for DI-10 and DI-15 solutions are close to those of DI-55, indicating suppression of the fluctuation effects.

Bates et al. ${ }^{14}$ and Jin and Lodge ${ }^{15}$ studied fluctuation effects on $G^{\prime}$ and $G^{\prime \prime}$ for diblock copolymer melts and high molecular weight diblock copolymer solutions in the disordered states, respectively. They reported that $G^{\prime}$ and $G^{\prime \prime}$ become almost one order and about 30\% higher than the respective (estimated) non-fluctuating values near the ODT. They pointed out that the observed terminal region behaviors are

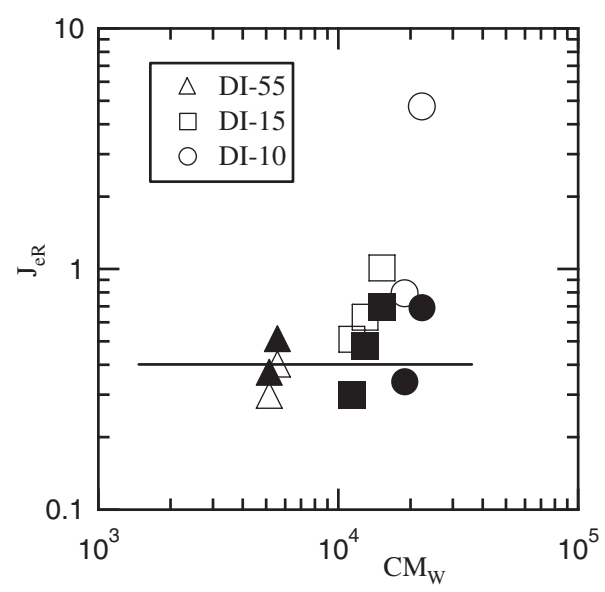

Figure 5. Double logarithmic plots of $J_{\mathrm{eR}} v s . C M$ for DI/DOP solutions obtained under oscillatory (open symbols) and steady (closed symbols) shear flows. Solid lines denote the values for homopolymers. Symbols are denoted in the figure.

qualitatively consistent with but higher than the theoretical calculation of Fredrickson and Larson, ${ }^{23}$ in which slowing down of the longest relaxation time due to the potential barrier created by fluctuating pattern similar with microphase separated structure in the ordered states is taken into account. Since the tested $\omega$ range in this study is limited in a relatively lower range, no obvious shifts of $G^{\prime}$ and $G^{\prime \prime}$ (appearance of shoulder for $G^{\prime}$ ) reported for diblock melts ${ }^{14}$ is observed in this study. However, it is clear that the degree of enhancements observed for $\eta^{0}$ and $J_{\mathrm{e}}$ are qualitatively consistent with above studies.

In the case of SPs and DPs, ${ }^{4}$ the fluctuation effects was only observed for $J_{\mathrm{e}}$ but not for $\eta^{0}$ at the similar values of $(\chi N)_{\text {eff }}$. We speculate that the difference in two different block copolymers can be attributed to the differences in the viscoelastic properties of components; since the viscoelastic properties of components are almost the same for SPs and DPs, existing fluctuation patterns have almost uniform viscoelastic proerties so that the barrier may become weaker in a dynamic scenes for SPs and DPs than for other diblock copolymers.

One may expect that $\eta^{0}$ obtained by oscillatory and steady shear flow measurements become the same since both data clearly show linear region behavior (Figure 3), however small differences are observed between the data near the ODT. Theoretical study has been further developed by Onuki, ${ }^{24}$ in which steady shear flow behaviors at finite $\dot{\gamma}$ are included. It was pointed out that proportionality factor for $\dot{\gamma}$ arising from deformation of fluctuations are different for viscosity and $N_{1}$ so that viscosity enhancements rapidly decrease even in the linear region for bare viscoelastic properties of copolymers. As a result, the Cox-Mertz law $^{25}$ does not hold for diblocks in 


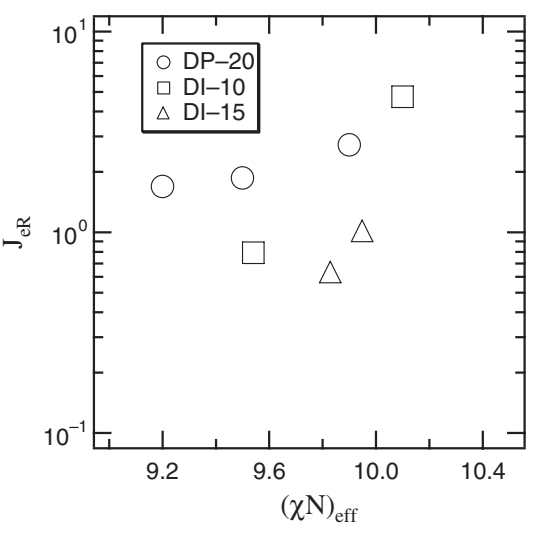

Figure 6. Semilogarithmic plots of $J_{\mathrm{eR}} v s .(\chi N)_{\mathrm{eff}}$ for DP-20, DI-10, and DI-15 solutions. Symbols are denoted in the figure.

the disordered states near the ODT. Above result for viscosity behavior is qualitatively consistent with this prediction.

Finally, we compare the $J_{\mathrm{eR}}$ data for different diblock copolymers. Figure 6 shows semilogarithmic plots of $J_{\mathrm{eR}} v s .(\chi N)_{\mathrm{eff}}$ for DP-20, DI-10, and DI-15. The data are scarce and somewhat scattered but we can point out that $J_{\mathrm{eR}}$ gradually increase with increase of $(\chi N)_{\text {eff }}$. There is no apparent difference between two samples in contrast to the viscosity behavior.

From above results, we conclude that the viscosity enhancement due to the fluctuation effects slightly depend on component viscosities; when viscosity ratio of components is close to 1 , the viscosity enhancement is negligible. On the other hand, enhancement of $J_{\mathrm{eR}}$ seems to be insensitive to the viscosity ratio of components.

\section{CONCLUSION}

Viscoelastic properties of poly(styrene- $\mathrm{d}_{8}-$ blockisoprene)s in DOP are measured under oscillatory and steady shear flow for three different molecular weight samples in the disordered states. Flow-induced microphase separation is not observed when effective interaction parameter $(\chi N)_{\text {eff }}$ is lower than 9.9. Enhancement of $\eta^{0}$ and $J_{\mathrm{e}}$ due to the fluctuation effects, which is more obvious for $J_{\mathrm{e}}$, is observed in oscillatory flow while suppression of fluctuation effects is observed in steady shear flow, confirmed by flow-SANS measurements. Comparing these results with those for poly(styrene-block-2-vinylpyridine)s, it is concluded that the viscosity enhancement due to the fluctuation effects slightly depend on component viscosities. When viscosity ratio of components is close to 1 , the viscosity enhancement is negligible. On the other hand, enhancement of $J_{\mathrm{eR}}$ seems to be insensitive to the viscosity ratio of components.

\section{REFERENCES}

1. Y. Takahashi, M. Noda, N. Ochiai, and I. Noda, Polymer, 37, 5943 (1996).

2. S. Kitade, N. Ochiai, Y. Takahashi, I. Noda, Y. Matsushita, A. Karim, A. I. Nakatani, H. Kim, and C. C. Han, Macromolecules, 31, 8083 (1998).

3. Y. Takahashi, M. Noda, S. Kitade, and I. Noda, J. Phys. Chem. Solids, 60, 1343 (1999).

4. Y. Takahashi, M. Noda, S. Kitade, K. Matsuoka, Y. Matsushita, and I. Noda, Polym. J., 37, 894 (2005).

5. Y. Takahashi, M. Naruse, Y. Akazawa, A. Takano, and Y. Matsushita, Polym. J., 37, 900 (2005).

6. Y. Takahashi, N. Ochiai, Y. Matsushita, and I. Noda, Polym. J., 28, 1065 (1996).

7. H. Watanabe, in "Rheology of Multiphase Polymeric Systems," T. Araki, T. C. Qui, and M. Shibayama, Ed., Marcel Dekker, New York, 1998, Chapter 9.

8. I. W. Hamley, Curr. Opin. Colloid Interface Sci., 5, 342 (2000).

9. K. A. Koppi, M. Tirrell, and F. S. Bates, Phys. Rev. Lett., 70, 1449 (1993).

10. K. I. Winey, S. S. Patel, R. G. Larson, and H. Watanabe, Macromolecules, 26, 2542 (1993).

11. K. I. Winey, S. S. Patel, R. G. Larson, and H. Watanabe, Macromolecules, 26, 4373 (1993).

12. N. P. Balsara, B. Hammouda, P. K. Kesani, S. V. Jonnalagadda, and G. C. Straty, Macromolecules, 27, 2566 (1994).

13. N. P. Balsara, H. Dai, P. K. Kesani, B. A. Garetz, and B. Hammouda, Macromolecules, 27, 7406 (1994).

14. J. H. Rhosedale and F. S. Bates, Macromolecules, 23, 2329 (1990).

15. X. Jin and T. P. Lodge, Rheol. Acta, 36, 229 (1997).

16. Y. Takahashi, M. Noda, M. Naruse, T. Kanaya, H. Watanabe, T. Kato, M. Imai, and Y. Matsushita, Nihon Reoroji Gakkaishi, 28, 187 (2000).

17. L. Leibler, Macromolecules, 13, 1602 (1980).

18. Y. Takahashi, S. Kitade, M. Noda, N. Ochiai, I. Noda, M. Imai, and Y. Matsushita, Polym. J., 30, 388 (1998).

19. Y. Takahashi, Y. Isono, I. Noda, and M. Nagasawa, Macromolecules, 18, 1002 (1985).

20. Y. Takahashi, M. Yamaguchi, D. Sakakura, and I. Noda, Nihon Reoroji Gakkaishi, 19, 39 (1991).

21. M. Yamaguchi, N. Maeda, Y. Takahashi, Y. Matsushita, and I. Noda, Polym. J., 23, 227 (1991).

22. W. W. Graessley, Adv. Polym. Sci., 16, 1 (1974).

23. G. H. Fredrickson and R. G. Larson, J. Chem. Phys., 86, 1553 (1987).

24. A. Onuki, J. Chem. Phys., 87, 3692 (1987).

25. W. P. Cox and E. H. Merz, J. Polym. Sci., 28, 619 (1958). 